\title{
Plant Defense against Necrotrophic Pathogens
}

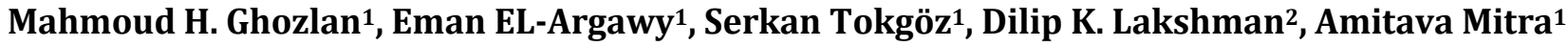 \\ ${ }^{1}$ University of Nebraska, Lincoln, NE, USA \\ ${ }^{2}$ USDA-ARS, Beltsville, MD, USA \\ Email:amitra1@unl.edu
}

How to cite this paper: Ghozlan, M.H., EL-Argawy, E., Tokgöz, S., Lakshman, D.K. and Mitra, A. (2020) Plant Defense against Necrotrophic Pathogens. American Journal of Plant Sciences, 11, 2122-2138. https://doi.org/10.4236/ajps.2020.1112149

Received: November 7, 2020

Accepted: December 26, 2020

Published: December 29, 2020

Copyright $\odot 2020$ by author(s) and Scientific Research Publishing Inc. This work is licensed under the Creative Commons Attribution International License (CC BY 4.0).

http://creativecommons.org/licenses/by/4.0/

\begin{abstract}
Necrotrophic pathogenic bacteria, fungi and oomycetes are widely distributed and are responsible for significant crop losses. Host plants deploy different defense mechanisms and appropriate immune responses to defend them against these pathogens. Regardless of the pathogen's lifestyle, infection activates plant immune responses either through Pathogen/Microbe Associated Molecular Pattern (P/MAMP) or through Effector Triggered Immunity (ETI). However, as R-genes are not usually associated with resistance to necrotrophs, resistance is largely dependent on the balanced interplay between crucial phytohormones in complex signaling pathways involving jasmonic acid (JA), ethylene, salicylic acid (SA) and abscisic acid (ABA). An increase in salicylic acid levels enhances susceptibility to necrotrophic pathogens but promotes resistance to hemibiotrophs, whereas a deficiency in SA or SA signaling has either no significant impact or affects resistance only at the primary infection site. The same fashion is observed for JA signaling system that appears to elicit resistance against diseases caused by necrotrophic pathogens and can trigger systemic immunity conferring resistance against them. On the other hand, $\mathrm{ABA}$ can play a positive or negative role in plant defense responses to necrotrophs as $\mathrm{ABA}$-mediated defense responses are dependent on specific plant-pathogen interactions. Understanding plant immune response against necrotrophic pathogens may lead to the development of resistant or tolerant crop cultivars.
\end{abstract}

\section{Keywords}

Effectors, Host Immunity, Signaling, P/MAMP, ETI, Disease Resistance

\section{Introduction}

Plants share their habitats with a plethora of pathogens but still the occurrence of disease is an exception. This is due to the plant's remarkable ability to recog- 
nize a potential pathogen and employ appropriate immune responses to defend itself. The invading pathogens can be classified on the basis of their mode of nutrition acquisition as necrotrophs, biotrophs or hemibiotrophs. Necrotrophic pathogens kill the host cells and then uptake nutrients; in contrast, biotrophs derive nutrients while keeping the host alive. Hemibiotrophs have both biotrophic and necrotrophic phases. They initially behave as biotrophs and then switch to the necrotrophic lifestyle, but the duration of the two phases varies from one hemibiotroph to another. Necrotrophs and biotrophs not only differ in their nutrient uptake strategies, but also in their virulence strategies and the disease symptoms they cause. Hence, the host has to deploy different defense mechanisms against pathogens with contrasting lifestyles. However, overlapping immune responses with interlinked genetic regulators also exists regardless of the lifestyle of the attacking pathogen [1].

Although plant resistance mechanisms against biotrophs have been investigated thoroughly, relatively less is understood about the same against necrotrophs [2]. As necrotrophic pathogens cause severe economic losses to the agricultural industry, it is important to develop effective strategies to control diseases caused by them. For this, in depth knowledge on the mechanism of pathogen virulence and plant immune responses is required [3] [4] [5].

\section{Necrotrophic Infection}

Necrotrophic pathogens can be bacteria, fungi or oomycetes. Infection generally involves host pathogen contact, secretion of phytotoxins and cell wall degrading enzymes (CWDEs), host penetration, primary lesion formation, lesion expansion, tissue maceration and death of the susceptible plant [6].

\subsection{Pathogen Entry}

The plant cell wall covered with cuticle forms the very first and important element of defense that prevents pathogen penetration. However, to invade a plant, the pathogen must enter. This can be done either by directly penetrating the plant by secreting hydrolytic enzymes or by entering through natural openings like stomata, or physical injuries. While bacteria use the latter method and inhabit the apoplast, fungi can penetrate either way. For direct entry into the plant, necrotic fungi use modified hyphae as infection structures. A specialized infection structure called appressorium helps the fungus make its way through the cell wall via a penetration peg. The penetration process is a result of the high turgor pressure developed inside the appressorium and the released hydrolytic enzymes, such as cutinases and CWDEs. The susceptibility of the host cell wall to degradation by CWDEs is related with the severity of necrotrophic infection [7].

\subsection{Plant Defense}

Regardless of the pathogen's lifestyle, pathogens infection activates plant immune 
responses in defense. Plant innate immunity consists of two intertwined branches: 1) Pathogen/Microbe Associated Molecular Pattern (P/MAMP) - Triggered Immunity or PTI and 2) Effector Triggered Immunity or ETI. These responses extensively share downstream signaling machinery but differences between the two lie in the nature of pathogen-derived molecules recognized and the intensity of the immune responses (Figure 1) [8].

\subsection{PAMP Triggered Immunity (PTI)}

Plants are able to recognize pathogens by identifying their slowly evolving molecular stamp called pathogen-associated molecular patterns (PAMPs), for example, fungal chitin and bacterial flagellin. They also recognize degradation products of microbial activity on plant cells, called damage-associated molecular patterns (DAMPs), such as plant cell wall-derived oligogalacturonides (OGs). The perception of these P/DAMPs via corresponding Pathogen Recognition Receptors (PRRs) leads to PAMP Triggered Immunity (PTI) [9] [10]. PRRs that have been characterized in association with immune responses to necrotrophic pathogens include membrane-localized receptor-like kinases (RLKs), like the chitin receptor LysM/CERK1 and the OG receptor WALL-ASSOCIATED KINASE 1 (WAK1) [11]. Typical RLKs consist of three regions: an extracellular

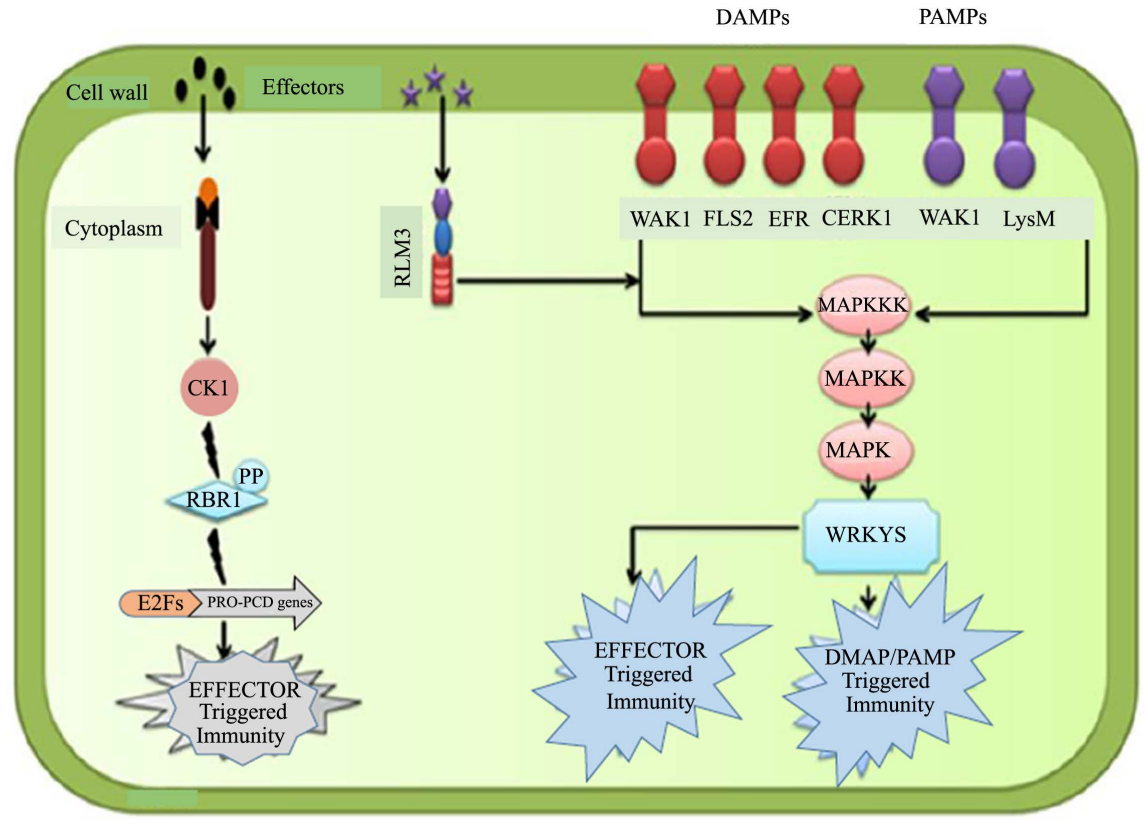

Figure 1. Different P/DAMPs, effectors, can initiate immune responses to necrotrophic pathogens. The recognition of D/PAMPs and effectors activates the basal immune responses through MAP kinase cascade. Effector-triggered $\mathrm{PCD} /$ susceptibility is initiated by NB-LRR-mediated perception of specific effectors secreted by pathogens. Effectors can trigger plant susceptibility in infected plants in a sequential manner starting after the recognition of the effectors by NB-LRR receptors then cyclin-dependent kinase inhibitors (CKIs) elicit the retinoblastoma-related 1 (RBR1) hyperphosphorylation which results in activation of programmed cell death genes and finally cytoplasmic shrinkage and leakage of ions and release of damage related molecular patterns molecules. 
N-terminal domain, commonly a leucine-rich repeat (LRR), a transmembrane domain and a cytosolic C-terminal Ser/Thr kinase domain. The variable LRR domain detects P/DAMPs, and the Ser/Thr domain signals defense cascades like mitogen-activated protein (MAP) kinase cascades. Distinct plant receptors perceive different elicitors, but the downstream responses converge into a common regulatory point to induce PTI.

The enzymes, polygalacturonases (PGs), secreted by necrotrophs during early stages of infections target the homogalacturonan component of pectin, releasing OGs of varying chain length. These OGs serve as DAMPS and are recognized by the extracellular domain of WAK1 that activates the kinase domain of the elongation factor $\mathrm{Tu}$ (EF-TU). This induces typical PTI responses, such as oxidative burst, cell wall lignifications, accumulation of phytoalexin, protease inhibitor expression, changes in ion fluxes, hormone biosynthesis and provides resistance to Botrytis cinerea in Arabidopsis [1]. Botrytis-induced kinase 1 (BIK1) is an early induced gene during infection of Arabidopsis by $B$. cinerea. It encodes a receptor-like cytoplasmic kinase (RLCK) which is a subclass of RLKs that lacks the extracellular domain. Loss of function mutant phenotypes of BIK1 highlights its requirement for resistance to fungal necrotrophic pathogens and its contrasting role as a defense suppressor against the hemibiotroph Pseudomonas syringae. BIK1 integrates the PTI responses from multiple PRRs like FLS2, EFR, and CERK1/LysM and is a major component in PTI signaling in Arabidopsis. It is also required for salicylic acid (SA) accumulation, ethylene (ET) signaling, and plant growth responses. BIK1 mutants clearly show the versatile roles of this immune response regulator and the interconnectivity of pathways in PTI responses to different pathogens [1]. Early stages of almost any infection share some common attributes like production of various secondary metabolites and hormones, host cell death, accumulation of reactive oxygen species (ROS), callose, and various cell wall modifications.

However, their relative abundance, timing and roles in host resistance or susceptibility depend on the lifestyle of the pathogen. For example, accumulation of ROS, such as superoxide anion and hydrogen peroxide is a ubiquitous immune response having contrasting defense functions depending on a pathogen's lifestyle. ROS molecules have diverse functions; they exert antimicrobial action indirectly by strengthening cell wall through oxidative cross linkage of glycoproteins, induce cellular signaling pathway like synthesis of salicylic acid, activate MAPK cascade and Systemic Acquired Resistance (SAR), and also cause cell death. At early stages of infection, the role of ROS in providing resistance to biotrophs and necrotrophs may be similar [12], interestingly during the later stages, plant-produced ROS confer resistance to biotrophs and hemibiotrophs through the regulation of cell death, but act as a virulence factor for necrotrophs. Studies show that aggressiveness of $B$. cinerea isolates correlates with the amount of $\mathrm{H}_{2} \mathrm{O}_{2}$ and $\mathrm{OH}^{-}$radicals present in the leaf tissue during infection. The fungus is able to tolerate the oxidative burst and produce ROS by itself. The Arabidopsis pentatricopeptide repeat protein PGN provides resistance to $B$. cinerea by regu- 
lating ROS homeostasis in mitochondria. Secondary metabolites are important chemical agents produced in defense to necrotrophs. These metabolites can be generated from pre-existing constituents (phytoanticipins), or synthesized de novo in response to pathogen intrusion (phytoalexins). Secondary metabolites implicated in defense against necrotrophs include derivatives of indole compounds: glucosinolates, phenylpropanoids, fatty acids, and flavanoids. The indole compounds, glucosinolates, and their derivatives are constitutively present in plant tissue, located in sulfur rich cells adjacent to myrosinases containing cells. Upon tissue damage, glucosinolates come in contact with myrosinases and get hydrolyzed to generate antimicrobial derivatives. In addition to serving as a passive defense, glucosinolates also participate in induced responses. Recent studies have shown that a PEN2-dependent glucosinolate metabolism pathway functions in broad-spectrum antifungal defense. PEN2 acts as a myrosinase that hydrolyzes glucosinolates and allows a directed movement of derivatives to sites of fungal penetration. This pathway is required for resistance to Plectosphaerella cucumerina and overlaps with PTI.

Arabidopsis accumulates many classes of pathogenesis-related proteins (PR) in response to infection like antifungal proteins, protease inhibitors, defensins, and other small peptides. Plant infection results in the expression of overlapping sets of PR-genes independent of the infecting pathogen. Plant defensins (PR-12) are small cysteine rich peptides stabilized by disulfide bonds which exhibit "cationic charges at physiological pH" [6]. In Arabidopsis, defensins are encoded by a multigenic family encoding 11 different defensins including the PDF1.2 genes. The exact mechanism of fungal inhibition by these proteins is not understood, but suggested mechanisms include disrupting calcium ion concentrations (required for hyphal tip growth), inducing ion-permeable pore formation in the fungal membrane, and effecting cytosolic targets. Mutations disrupting JA- and ET-defense responses abrogate PDF1.2 expression and resistance to many necrotrophic fungi. Although, the role of PDF1.2 in Arabidopsis defense is unclear, the mutant data shows it definitely has a function in resistance to necrotrophic fungi.

\subsection{Effector Triggered Immunity (ETI)}

Virulent pathogens deploy effectors that interfere with PTI [13]. Direct or indirect effector recognition by R-proteins, usually nucleotide-binding site leucine-rich repeat (NBS-LRR) proteins, initiates Effector Triggered Immunity (ETI) in biotrophs, which is a more robust immune response. However, no R-gene has been associated with resistance to necrotrophs except Arabidopsis resistance to Leptosphaeria maculans 3 (RLM3), a Toll/interleukin 1 receptor domain R-protein which provides broad immunity against several necrotrophs. In contrast to biotrophs, R-gene mediated response to some Host Specific Necrotrophs (HSNs) lead to host susceptibility [14]. A gene-for-gene relationship between Host Specific Toxins (HSTs) and the host R-protein results in disease that can be termed as Effector-Triggered Susceptibility (ETS). For example, the 
HSN fungus Pyrenophora tritici-repentis releases ToxA toxins that indirectly interact with the R-proteins encoded by Tsn1, the wheat toxin sensitivity gene, and lead to wheat $\tan$ spot disease. Similarly, nonspecific toxins produced by broad host-range necrotrophs (BHNs) target host proteins to promote infection. Necrotrophic fungi and bacteria produce necrosis inducing proteins (NEPs) which cause cell death in dicots. This suggests that necrotrophs exploit plant immune responses for virulence. Just like the PTI response, downstream ETI response events also activate the MAPK cascade and WRKY transcription factors. This induces the transcription of pathogenesis-related (PR) genes in and around the infected cell for the production of salicylic acid (SA), jasmonic acid (JA), ethylene (ET), antimicrobial compounds and cell wall strengthening [7].

\subsection{Cell Death during Necrotrophic Infection}

The hypersensitive response (HR) is a cell death induced at the site of infection by biotrophic pathogens, which indicates resistance because it confines pathogens by abolishing nutrient supply. Conversely, cell death occurring during infection by necrotrophs is an indicator of successful infection as activation of cell death enhances colonization by necrotrophic pathogens. Consequently, biotrophs actively suppress HR cell death, whereas necrotrophs promote cell death by deploying diverse virulence factors [15]. When HR cell death is induced by pathogens, the plant cell morphology changes drastically which includes chromatin condensation, cytoplasmic shrinkage, mitochondrial swelling, vacuolization and chloroplast disruption [11]. However, effector stimulated cell death is not fairly understood. It is presumed that signaling modules regulate NB-LRRs, the effector receptors, which integrate redox signals downstream of NADPH oxidase leading to SA accumulation. As a result, SA and ROS act synergistically to drive HR [12] [16].

Sclerotinia sclerotiorum secretes an effector, SsCP, that interacts with pathogenesis-related protein 1 (PR-1) and plays an important role in host colonization [17]. Several effectors from Rhizoctonia solani, namely, AGLIP1, RsLysM and RSAG8_06778 have also been shown to induce host cell death [18] [19] [20]. Arabidopsis plants impaired in genes encoding caspase-like enzymes, vacuolar processing enzymes (VPE) and metacaspases (MCA) differ in their susceptibility to necrotrophs. Knock-out mutants of type II metacaspases-mca2, mca3, mca4, mca5 and mca6 exhibited reduced cell death in reaction to Botrytis, whereas knock-out mutants of type I-mca7 and mca8 showed stronger symptoms of cell death. The Arabidopsis knock-out mutant studies also show that VPEs trigger vacuole membrane disruption, DNA fragmentation and finally cell death [20].

\subsection{Plant Signaling Network}

Plant responses to pathogens have been studied deeply with respect to plant hormone synthesis and signaling. Changes in hormonal homeostasis promote either resistance or susceptibility by creating a range of pathological conditions exploited by pathogens. Resistance to necrotrophs is largely dependent on com- 
plex signaling pathways involving JA, ET and abscisic acid (ABA). Defense against necrotrophs is highly dependent on the balanced interplay between these crucial phytohormones, which may act differently in different pathosystems [21].

\subsection{SA-Dependent Signaling Pathway}

The function of SA in immune responses varies among plant species. An increase in SA levels enhances susceptibility to necrotrophic pathogens but promotes resistance to hemibiotrophs, whereas a deficiency in SA or SA signaling has either no significant impact or affects resistance only at the primary infection site. Although, SA is dispensable for flagellin-induced resistance to $B$. cinerea, the pathogen's toxin and virulence factor botrydial elicits HR-like cell death early during infection in an SA-dependent manner. It has been observed that Arabidopsis and tobacco plants defective in SA signaling are more insensitive to botrydial than wild-type plants, as opposed to plants defective in JA signaling, which are more sensitive. Similarly, $B$. cinerea secretes a virulence factor $\beta$-(1,3)(1,6)-D-glucan, an exopolysaccharide (EP) that activates the SA pathway, which works antagonistically to the JA pathway, and hence, enhances disease severity in tomato. Thus, $B$. cinerea exploits the SA pathway to establish disease in tomato and Arabidopsis [22]. Virulent necrotrophic pathogens have to defeat the immune responses induced by SA signaling system to cause disease. Necrotrophic pathogens produce effectors to suppress the plant immune responses.

The effector proteins usually target basic innate immunity in plants [12] [23]. The bacterial effector Hopl1 expression in Arabidopsis plant cells generate a 60\% decrease in the level of SA-inducible PR1 gene transcript and about 50\% lower free and total SA levels, which may indicate that Hopl1 suppresses SA accumulation and SA-dependent defenses [24]. Similarly, Coronatine a bacterial toxin produced by Pseudomonas syringae was reported to activate three homologous NAC transcription factor (TF) genes through direct activity of the transcription factor MYC2. The elicited NAC TFs inhibit the isochorismate synthase 1 (ICS1) gene that is involved in SA biosynthesis. These TFs also activate expression of BSMT1 gene encoding SA methyltransferase, which reforms SA to the inactive volatile methyl SA (MeSA). These findings point to a clue that the toxin produced by the bacterial pathogen promotes systemic susceptibility by suppressing SA signaling [25]. Coronatine can mimic jasmonic acid-isoleucine and induce bacterial entry, bacterial growth in the apoplast, and systemic susceptibility.

\subsection{JA/ET-Dependent Signaling Pathway}

JA signaling system appears to elicit resistance against diseases caused by necrotrophic pathogens and can trigger systemic immunity conferring resistance against them. [26] [27] [28] [29] [30]. JA signaling systems modulate plant immune responses and confer resistance or susceptibility against different pathogens [31] [32]. The defense function of JA is common to necrotrophic fungi and insect pests. Arabidopsis JA-knockout mutants display increased susceptibility 
to several Botrytis species, for example, jar1 with impaired JA signaling shows enhanced susceptibility to $B$. cinerea. Additionally, JA level is substantially increased in Alternaria brassicicola challenged plants. The tga256 (the TGA transcription factors TGA2, TGA5 and TGA6) triple mutant impaired in JA-induced PDF1.2 and b-CHI gene expression showed higher susceptibility against $B$. cinerea [33]. These results suggest that intact JA signaling system is necessary to induce resistance against the necrotrophic pathogen $B$. cinerea. The mediator complex subunit 25 induces expression of JA-dependent defense genes and triggers resistance to the necrotrophic fungal pathogens $A$. brassicicola and $B$. cinerea in Arabidopsis [34] [35]. All together these studies suggest that JA signaling is affected the triggering of resistance against necrotrophic pathogens (Figure 2).

Ethylene (ET) signaling system is an important component in plant innate immune system and plays an important role in defense against necrotrophs [36] [37]. Pathogen invasion or PAMP recognition results in elevated expression of ethylene biosynthetic genes leading to enhanced ethylene biosynthesis [38] [39] [40]. Ethylene mutation in ethylene signaling in Arabidopsis does not influence resistance to A. brassicicola, but compromises Arabidopsis defense against Botrytis. Moreover, Arabidopsis ein 2 - 5 (ethylene insensitive) mutant shows susceptibility to $F$. oxysporum and $S$. sclerotiorum [41]. Ethylene may act as a two-faceted player in the plant immune response network, triggering resistance or susceptibility against different pathogens [42] [43] [44]. Ethylene has been shown to be an integral part of PAMP-triggered immunity. Ethylene perception and signaling are crucial for the PRR FLS2 gene transcription [45].

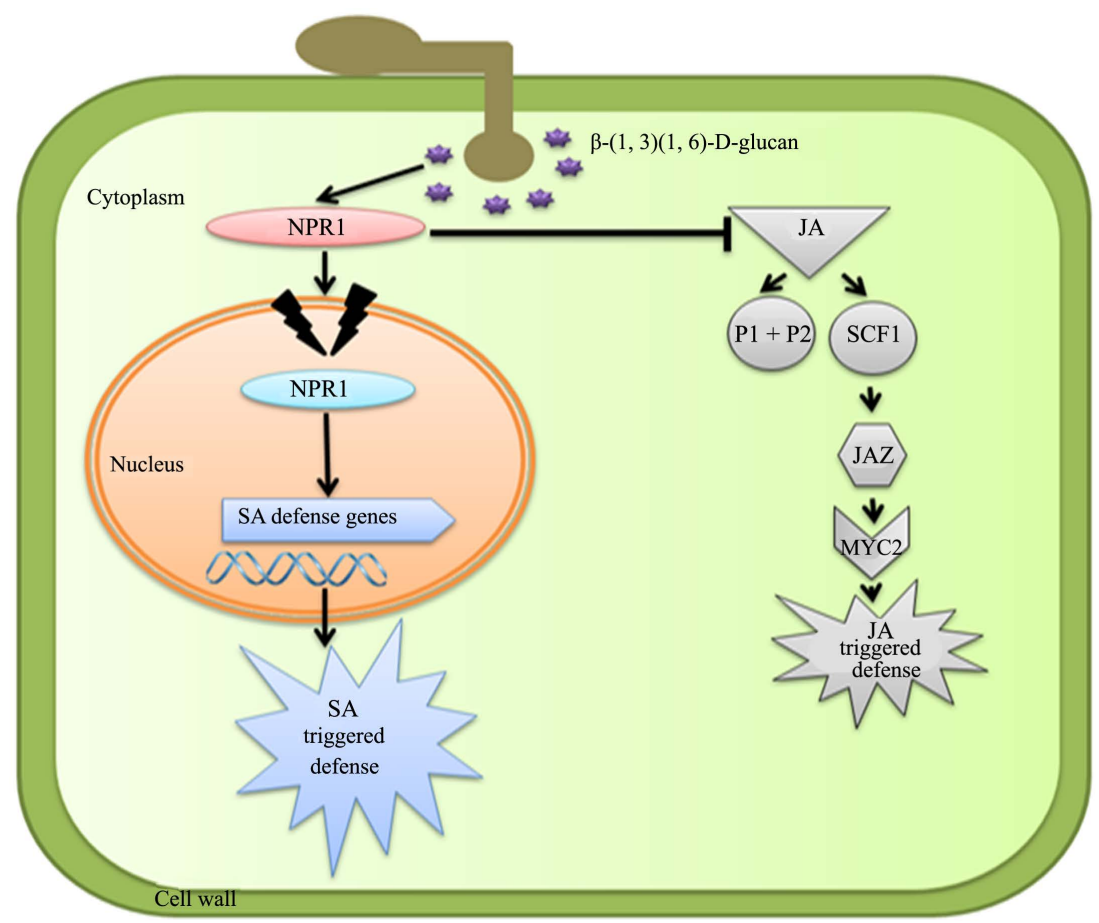

Figure 2. Botrytis cinerea secretes a virulence factor $\beta$ - $(1,3)(1,6)$-D-glucan that play a dual role in inducing SA related defense by suppressing JA signaling system to cause disease. 
JA and ET pathways appear to function cooperatively in modulating plant immune responses [28] [46]. JA and ET work synergistically in the induction of defense-related genes against necrotrophs. Both JA and ET signaling are required for the expression of the defense-related gene PDF1.2 [47] [48]. JA and ET signaling pathways mediate various defense responses, either independently or collaboratively. JA stimulates proteinase inhibitor gene expression while ET alone does not, but JA and ET induce proteinase inhibitor genes synergistically in tobacco [49]. ET positively regulates the induction of allene oxide synthase (AOS), which catalyzes the first step in the biosynthesis of JA, while MeJA induced a rapid increase in 1-aminocyclopropane-1-carboxylic acid oxidase (ACO), resulting in increased synthesis of ET [50]. These results suggest that ET induces JA biosynthesis, while JA triggers ET biosynthesis and both JA and ET signaling pathways are interlinked.

\subsection{ABA-Dependent Signaling Pathway}

Abscisic acid (ABA) is a plant hormone involved in immune signal transduction system. ABA can play a positive or negative role in plant defense responses to necrotrophs as ABA-mediated defense responses are dependent on specific plant-pathogen interactions. PAMP recognition activates ABA biosynthesis pathway and accumulation of $A B A$ in the infected tissues. Infection of Arabidopsis with Pythium irregulare leads to rapid increase in ABA levels and mutants impaired in ABA signaling display enhanced susceptibility to A. brassicicola, suggesting that $\mathrm{ABA}$ has a positive effect on defense against these pathogens. However, ABA-deficient mutants are resistant to B. cinerea and Fusarium oxysporum, indicating that $\mathrm{ABA}$ has a negative effect on defense against these pathogens [51]. ABA was rapidly synthesized in plants following inoculation with necrotrophic fungal pathogens and the concentration of $\mathrm{ABA}$ increased in sugar beet (Beta vulgaris) leaves during Cercospora beticola infection [24]. After an initial burst of $\mathrm{ABA}$ induced by inoculation, elevated $\mathrm{ABA}$ concentrations were detected during the fungal penetration and colonization phases [52]. The role of $\mathrm{ABA}$ in plant defense to necrotrophic pathogens has been suggested to be correlated to the stage of infection. It seems to play positive roles during the early events of infection by affecting stomatal closure and deposition of callose and the negative roles usually at later stages that include suppressing ROS accumulation and SA induction [53] [54]. It has also been suggested that the dual role of $\mathrm{ABA}$ inducing resistance and susceptibility may be due to the differential action of upstream and downstream components in ABA signaling pathway [55].

\subsection{SA/JA Cross-Talk}

What happens when plants are attacked by biotrophs and necrotrophs at the same time? How do they coordinate their defenses? Studies show that infection by a biotroph significantly reduces resistance against a necrotroph because SA works antagonistically to JA. However, cross-talks between these two hormones are more tightly controlled than expected. On attack by a biotrophic pathogen, 
tradeoff with necrotrophic resistance may be important to maintain basal resistance against the attacking biotroph. Antagonism between SA and JA signaling pathways has been widely reported in plants. Furthermore, SA production during pathogen infection suppresses JA biosynthesis [56] [57] [58] [59]. As JA signaling antagonizes SA signaling system, the Arabidopsis mutants which accumulate high levels of SA, show repression of JA-mediated pathway [60]. SA promotes disease development caused by necrotrophic pathogens by suppressing JA signaling system.

Lipoxygenase (LOX) is the key enzyme in the JA biosynthesis pathway. Pathogen-induced SA suppresses LOX2 gene in Arabidopsis leading to decrease in JA biosynthesis. Compared with wild-type plants, transgenic NahG plants showed enhanced expression of LOX2 and synthesized 25-fold higher levels of JA during pathogen infection. Leon-Reyes et al. [61] showed that the JA biosynthesis genes LOX2 encoding lipoxygenase, AOS encoding allene oxide synthase, AOC2 encoding allene oxide cyclase, and OPR3 encoding 12-oxo-phytodienoate reductase were all repressed by SA in Arabidopsis. Furthermore, SA strongly antagonized the JA signaling pathway, resulting in the downregulation of a large set of JA-responsive genes, including the marker genes PDF1.2 and JA-responsive genes (VSP2) in A. thaliana (Figure 3).

In the Arabidopsis aos/dde2 mutant, expression of the JA biosynthesis enzyme allene oxide synthase (AOS) is completely blocked. Mutant aos/dde2 plants did not express the JA-responsive marker genes PDF1.2 or VSP2 in response to infection with the necrotrophic fungus $A$. brassicicola. The expression of the JA-responsive genes VSP and PDF1.2 was enhanced strongly in the transgenic NahG gene expressing Arabidopsis plants that degraded SA [62]. The results suggest that in wild-type plants JA signaling is inhibited by SA that accumulates during pathogen infection. Moreover, SA/JA cross-talk is prevented during R-protein mediated programmed cell death (PCD). This spatial and pathogen

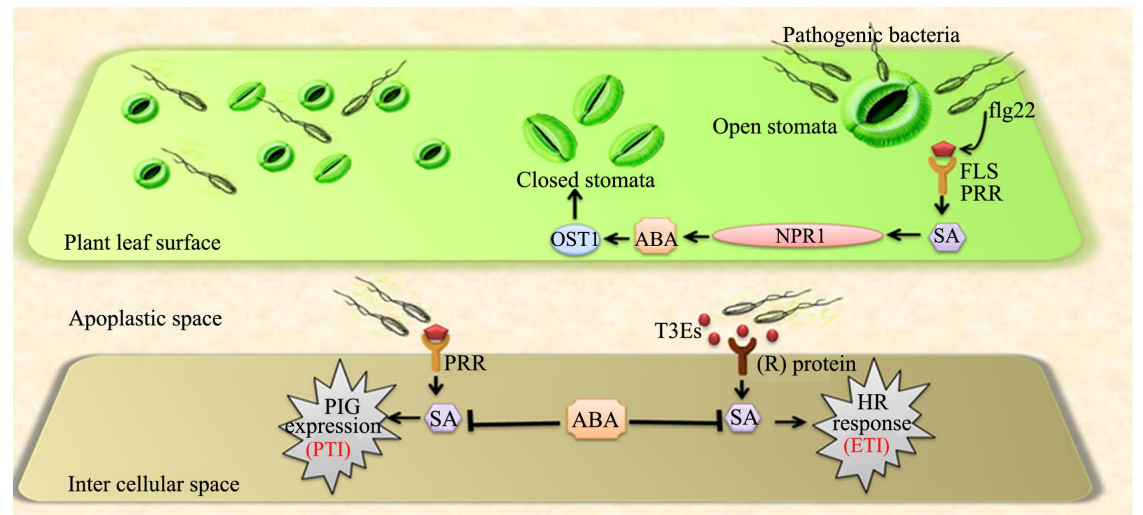

Figure 3. The dual role of ABA in immunity responses in the Arabidopsis thaliana-Pseudomonas syringae pathosystem. ABA plays a positive role in first stage stomatal immunity on leaf surface area by induction of stomatal closure to prevent pathogen entry. ABA also plays a negative role in second stage PAMP triggered immunity (PTI) and effector-triggered immunity (ETI). 
type-specific control of SA/JA cross-talk shows the ability of the plant immune system to cope with multiple pathogens [63] [64]. However, in some cases, SA does not inhibit JA biosynthesis and may act synergistically as concentrations of both phytohormones determine synergism or antagonism. Low concentrations favor synergistic actions, such as induction of Programmed Cell Death (PCD), whereas high concentrations cause antagonistic effects between JA and SA. SA treated leaves inoculated with $A$. brassicicola exhibited severe disease symptoms, which correlated with strong expression of PR-1 gene. Collectively these studies suggest that SA may suppress biosynthesis of JA, resulting in the suppression of JA signaling system (Figure 4).

\subsection{Regulation of Phytohormone Signaling by Transcription Factors and Chromatin Modifications}

PTI and ETI activate both local and systemic defense responses (called systemic acquired resistance $[S A R])$, which are modulated by plant hormones. These responses to pathogen attack require large-scale transcriptional reprogramming which include transcription factor (TF) families such as WRKY genes [8]. WRKY70 transcription factors positively regulate defense genes that are under control of SA and repress JA-dependent genes. The upregulation of Arabidopsis WRKY70 confers enhanced resistance to Erysiphe cichoracearum but impairs resistance to $A$. brassicicola. WRKY18, 40 and 60 are positive regulators of defense reactions against $B$. cinerea and negative regulators of defense against Pseudomonas syringae. This was shown by the knockout mutants of these three WRKY genes that had an opposite effect on the expression of SA-regulated PR-1 and JA-dependent PDF1.2 genes. Recent investigations have also identified

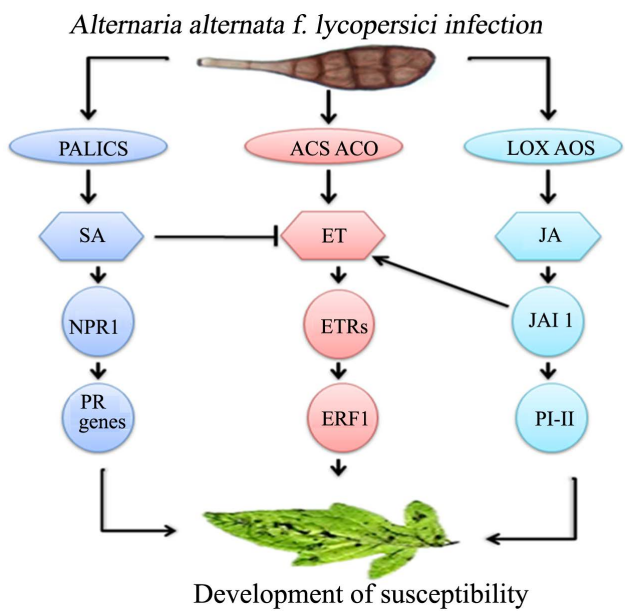

Figure 4. The role of phytohormone complex (ethylene, jasmonic acid and salicylic acid) in the development of disease susceptibility of $A$. alternate $\mathrm{f}$ sp. lycopersici infected tomato. Both the ET and JASMONIC ACID INSENSITIVE1 (JAI1) receptor-dependent JA signaling pathway are necessary for susceptibility, while SA response promotes resistance to infection. In addition, the role of JA in susceptibility is partly dependent on ET biosynthesis and perception, while the SA pathway increases resistance to infection and antagonizes the ET response. 
WRKY33 responsible for plant defense against necrotrophs. WRKY33 mutation caused enhanced susceptibility to $B$. cinerea and $A$. brassicicola but increased resistance to the virulent strain of $P$. syringae, which correlated with stronger activation of SA-regulated genes PR-1, PR-5 and PAD4 and reduced level of expression of JA-regulated gene, PDF1.2 [9] [41]. In addition to TFs, modifications of histones and chromatin-remodeling have a fundamental role in gene regulation and response to pathogen attack. The SWI/SNF chromatin-remodeling ATPase SPLAYED is required for defense against $B$. cinerea as it regulates the expression of several genes in the JA/ET-dependent defense pathway. Moreover, some necrotrophs produce toxins that interfere with plant chromatin-modification machinery to suppress defense [6].

\section{Exploiting Necrotrophic Effectors for Crop Protection}

All the knowledge gained on the mechanism of pathogen virulence and plant immune responses can be exploited for our benefit. As mentioned before, resistance to necrotrophs has been found to be partial and no R gene has been identified. Nevertheless, the virulence-promoting role of necrotrophic effectors and their targets can be used to develop cultivars that are more resistant. These cultivars can be further utilized in breeding for disease resistance. The identification and production of such effectors allow breeders to select introgressions that are insensitive to the effector, which can then be used to improve crop cultivars.

\section{Acknowledgements}

Supported in part by grants from the Nebraska Dry Bean Commission and Nebraska Department of Agriculture.

\section{Conflicts of Interest}

The authors declare no conflicts of interest regarding the publication of this paper.

\section{References}

[1] Mengiste, T. (2012) Plant Immunity to Necrotrophs. Annual Review of Phytopathology, 50, 267-294. https://doi.org/10.1146/annurev-phyto-081211-172955

[2] Spanu, P.D. and Panstruga, R. (2017) Editorial: Biotrophic Plant-Microbe Interactions. Frontiers in Plant Science, 8, 192. https://doi.org/10.3389/fpls.2017.00192 https://www.frontiersin.org/article/10.3389/fpls.2017.00192

[3] van Kan, J.A.L. (2006) Licensed to Kill: The Lifestyle of a Necrotrophic Plant Pathogen. Trends in Plant Science, 11, 247-253. https://doi.org/10.1016/j.tplants.2006.03.005

[4] Wei, M., Wang, A., Liu, Y., Ma, L., Niu, X. and Zheng, A. (2020) Identification of the Novel Effector RsIA_NP8 in Rhizoctonia solani AG1 IA That Induces Cell Death and Triggers Defense Responses in Non-Host Plants. Frontiers in Microbiology, 11, 1115. https://doi.org/10.3389/fmicb.2020.01115

[5] Pandey, D., Rajendran, S., Gaur, M., Sajeesh, P.K. and Kumar, A. (2016) Plant De- 
fense Signaling and Responses against Necrotrophic Fungal Pathogens. Journal of Plant Growth Regulation, 35, 1159-1174.

https://doi.org/10.1007/s00344-016-9600-7

[6] Laluk, K. and Mengiste, T. (2010) Necrotroph Attacks on Plants: Wanton Destruction or Covert Extortion? The Arabidopsis Book, 8, e0136.

https://doi.org/10.1199/tab.0136

[7] Muthamilarasan, M. and Prasad, M. (2013) Plant Innate Immunity: An Updated Insight into Defense Mechanism. Journal of Biosciences, 38, 433-449. https://doi.org/10.1007/s12038-013-9302-2

[8] Pandey, S.P. and Somssich, I.E. (2009) The Role of WRKY Transcription Factors in Plant Immunity. Plant Physiology, 150, 1648-1655.

https://doi.org/10.1104/pp.109.138990

[9] Łaźniewska, J., Macioszek, V.K., Lawrence, C.B. and Kononowicz, A.K. (2010) Fight to the Death: Arabidopsis thaliana Defense Response to Fungal Necrotrophic Pathogens. Acta Physiologiae Plantarum, 32, 1-10. https://doi.org/10.1007/s11738-009-0372-6

[10] Thomma, B.P.H.J., Nürnberger, T. and Joosten, M.H.A.J. (2011) Of PAMPs and Effectors: The Blurred PTIETI Dichotomy. The Plant Cell, 23, 4-15.

https://doi.org/10.1105/tpc.110.082602

[11] Gong, B.-Q., Wang, F.-Z. and Li, J.-F. (2020) Hide-and-Seek: Chitin-Triggered Plant Immunity and Fungal Counterstrategies. Trends in Plant Science, 25, 8. https://doi.org/10.1016/j.tplants.2020.03.006

[12] Wen, L. (2012) Cell Death in Plant Immune Response to Necrotrophs. Journal of Plant Biochemistry \& Physiology, 1, 1-3.

https://doi.org/10.4172/2329-9029.1000e103

[13] Jones, D.G.J. and Dangl, J.L. (2006) The Plant Immune System. Nature, 444, 323-329. https://doi.org/10.1038/nature05286

[14] Wang, X., Jiang, N., Liu, J., Liu, W. and Wang, G.L. (2014) The Role of Effectors and Host Immunity in Plant-Necrotrophic Fungal Interactions. Virulence, 5, 722-732. https://doi.org/10.4161/viru.29798

[15] Glazebrook, J. (2005) Contrasting Mechanisms of Defense against Biotrophic and Necrotrophic Pathogens. Annual Review of Phytopathology, 43, 205-227. https://doi.org/10.1146/annurev.phyto.43.040204.135923

[16] Coll, N.S., Epple, P. and Dangl, J.L. (2011) Programmed Cell Death in the Plant Immune System. Cell Death \& Differentiation, 18, 1247-1256.

https://doi.org/10.1038/cdd.2011.37

[17] Yang, G., Tang, L., Gong, Y., Xie, J., Fu, Y., Jiang, D., et al. (2018) A Cerato-Platanin Protein SsCP1 Targets Plant PR1 and Contributes to Virulence of Sclerotinia sclerotiorum. New Phytologist, 217, 739-755. https://doi.org/10.1111/nph.14842

[18] Li, S., Peng, X., Wang, Y., Hua, K. Y., Xing, F., Zheng, Y.Y., et al. (2019) The Effector AGLIP1 in Rhizoctonia solani AG1 IA Triggers Cell Death in Plants and Promotes Disease Development through Inhibiting PAMP-Triggered Immunity in Arabidopsis thaliana. Frontiers in Microbiology, 10, 2228. https://doi.org/10.3389/fmicb.2019.02228

[19] Dölfors, F., Holmquist, L., Dixelius, C. and Tzelepis, G. (2019) A LysM Effector Protein from the Basidiomycete Rhizoctonia solani Contributes to Virulence through Suppression of Chitin Triggered Immunity. Molecular Genetics and Genomics, 294, 1211-1218. https://doi.org/10.1007/s00438-019-01573-9 
[20] Anderson, J.P., Sperschneider, J., Win, J., Kidd, B., Yoshida, K., Hane, J., et al. (2017) Comparative Secretome Analysis of Rhizoctonia solani Isolates with Different Host Ranges Reveals Unique Secretomes and Cell Death Inducing Effectors. Scientific Reports, 7, Article No. 10410. https://doi.org/10.1038/s41598-017-10405-y

[21] Checker, V.G., Kushwaha, H.R., Kumari, P. and Yadav, S. (2018) Role of Phytohormones in Plant Defense: Signaling and Cross Talk. In: Singh, A. and Singh, I., Eds., Molecular Aspects of Plant-Pathogen Interaction, Springer, Singapore, 159-184. https://doi.org/10.1007/978-981-10-7371-7_7

[22] Caarls, L., Pieterse, C.M.J. and Van Wees, S.C.M. (2015) How Salicylic Acid Takes Transcriptional Control over Jasmonic Acid Signaling. Frontiers in Plant Science, 6, 170. https://www.frontiersin.org/article/10.3389/fpls.2015.00170 https://doi.org/10.3389/fpls.2015.00170

[23] Wu, Y., Zhang, D., Chu, J.Y., Boyle, P., Wang, Y., Brindle, I.D., De Luca, V. and Després, C. (2012) The Arabidopsis NPR1 Protein Is a Receptor to the Plant Defense Hormone Salicylic Acid. Cell Reports, 1, 639-647. https://doi.org/10.1016/j.celrep.2012.05.008

[24] Jelenska, J., Yao, N., Vinatzer, B.A., Wright, C.M., Brodsky, J.L. and Greenberg, J.T. (2007) AJ Domain Virulence Effector of Pseudomonas syringae Remodels Host Chloroplasts and Suppresses Defenses. Current Biology, 17, 499-508. https://doi.org/10.1016/j.cub.2007.02.028

[25] Zheng, X., Spivey, N.W., Zeng, W., Po-Pu, L., Fu, Z.Q., Klessig, D.F., He, S.Y. and Dong, X. (2012) Coronatine Promotes Pseudomonas syringae Virulence in Plants by Activating a Signaling Cascade That Inhibits Salicylic Acid Accumulation. Cell Host \& Microbe, 11, 587-596. https://doi.org/10.1016/j.chom.2012.04.014

[26] Bakker, P., Doornbos, R.F., Zamioudis, C., Berendsen, R.L. and Pieterse, C.M.J. (2013) Induced Systemic Resistance and the Rhizosphere Microbiome. The Plant Pathology Journal, 29, 136-143. https://doi.org/10.5423/PPJ.SI.07.2012.0111

[27] Kravchuk, Z., Vicedo, B., Flors, V., Camańes, G., González-Bosch, C. and Garcia-Agustin, P. (2011) Priming for JA-Dependent Defenses Using Hexanoic Acid Is an Effective Mechanism to Protect Arabidopsis against B. cinerea. Journal of Plant Physiology, 186, 359-366. https://doi.org/10.1016/j.jplph.2010.07.028

[28] Martinez-Medina, A., Fernández, I., Sánchez-Guzmán, M.J., Jung, S.C., Pascual, J.A. and Pozo, M.J. (2013) Deciphering the Hormonal Signalling Network behind the Systemic Resistance Induced by Trichoderma harzianum in Tomato. Frontiers in Plant Science, 4, 206. https://doi.org/10.3389/fpls.2013.00206

[29] Niu, Y., Figueroa, P. and Browse, J. (2011) Characterization of JAZ-Interacting bHLH Transcription Factors That Regulate Jasmonate Responses in Arabidopsis. Journal of Experimental Botany, 62, 2143-2154. https://doi.org/10.1093/jxb/erq408

[30] Weller, D.M., Mavrodi, D.V., Van Pelt, J.A., Pieterse, C.M.J., Van Loon, L.C. and Bakker, P.A.H.M. (2012) Induced Systemic Resistance (ISR) in Arabidopsis thaliana against Pseudomonas syringae pv. tomato by 2,4-Diacetylphloroglucinol-Producing Pseudomonas fluoresces. Phytopathology, 102, 403-412. https://doi.org/10.1094/PHYTO-08-11-0222

[31] Zamioudis, C. and Pieterse, C.M.J. (2012) Modulation of Host Immunity by Beneficial Microbes. Molecular Plant-Microbe Interactions, 25, 139-150. https://doi.org/10.1094/MPMI-06-11-0179

[32] Moffat, C.S., Ingle, R.A., Wathugala, D.L., Saunders, N.J., Knight, H. and Knight, M.R. (2012) ERF5 and ERF6 Play Redundant Roles as Positive Regulators of JA/Et-Mediated Defense against Botrytis cinerea in Arabidopsis. PLoS ONE, 7, 
e35995. https://doi.org/10.1371/journal.pone.0035995

[33] Zander, M., La Camera, S., Lamotte, O., Metraux, J.P. and Gatz, C. (2010) Arabidopsis thaliana Class-II TGA Transcription Factors Are Essential Activators of Jasmonic Acid/Ethylene-Induced Defense Responses. The Plant Journal: For Cell and Molecular Biology, 61, 200-210. https://doi.org/10.1111/j.1365-313X.2009.04044.x

[34] An, C. and Mou, Z. (2013) The Function of the Mediator Complex in Plant Immunity. Plant Signaling \& Behavior, 8, e23182. https://doi.org/10.4161/psb.23182

[35] Kidd, B.N., Edgar, C.I., Kumar, K.K., Aitken, E.A., Schenk, P.M., Manners, J.M. and Kazan, K. (2009) The Mediator Complex Subunit PFT1 Is a Key Regulator of Jasmonate-Dependent Defense in Arabidopsis. Plant Cell, 21, 2237-2252. https://doi.org/10.1105/tpc.109.066910

[36] Berr, A., McCallum, E.J., Alioua, A., Heintz, D., Heitz, T. and Shen, W.H. (2010) Arabidopsis Histone Methyltransferase SET Domain Group 8 Mediates Induction of the Jasmonate/Ethylene Pathway Genes in Plant Defense Response to Necrotrophic Fungi. Plant Physiology, 154, 1403-1414.

https://doi.org/10.1104/pp.110.161497

[37] Shakeel, S.N., Wang, X., Binder, B.M. and Schaller, G.E. (2013) Mechanisms of Signal Transduction by Ethylene: Overlapping and Non-Overlapping Signaling Roles in a Receptor Family. AoB Plants, 5, plt010. https://doi.org/10.1093/aobpla/plt010

[38] Denoux, C., Galletti, R., Mammarella, N., Gopalan, S., Werck, D., De Lorenzo, G., Ferrari, S., Ausubel, F.M. and Dewdney, J. (2008) Activation of Defense Response Pathways by OGs and Flg22 Elicitors in Arabidopsis Seedlings. Molecular Plant, 1, 423-445. https://doi.org/10.1093/mp/ssn019

[39] Nambeesan, S., AbuQamar, S., Laluk, K., Mattoo, A.K., Mickelbart, M.V., Ferruzzi, M.G., Mengiste, T. and Handa, A.K. (2012) Polyamines Attenuate Ethylene-Mediated Defense Responses to Abrogate Resistance to Botrytis cinerea in Tomato. Plant Phy siology, 158, 1034-1045. https://doi.org/10.1104/pp.111.188698

[40] Vidhyasekaran, P. (2014) PAMP Signals in Plant Innate Immunity: Signal Perception and Transduction. Springer, Dordrecht, 442.

https://doi.org/10.1007/978-94-007-7426-1

[41] Zhu, L., Li, Y., Li, L., Yang, J. and Zhang, M. (2011) Ethylene Is Involved in Leafy Mustard Systemic Resistance to Turnip Mosaic Virus Infection through the Mitochondrial Alternative Oxidase Pathway. Physiological and Molecular Plant Pathology, 76, 166-172. https://doi.org/10.1016/j.pmpp.2011.09.005

[42] Akagi, A., Dandekar, A.M. and Stotz, H.U. (2011) Resistance of Malus domestica Fruit to Botrytis cinerea Depends on Endogenous Ethylene Biosynthesis. Phytopathology, 101, 1311-1321. https://doi.org/10.1094/PHYTO-03-11-0087

[43] Son, G.H., Wan, J., Kim, H.J., Nguyen, X.C., Chung, W.-S., Hong, J.C. and Stacey, G. (2012) Ethylene-Responsive Element-Binding Factor 5, ERF5, Is Involved in Chitin-Induced Innate Immunity Response. Molecular Plant-Microbe Interactions, 25, 48-60. https://doi.org/10.1094/MPMI-06-11-0165

[44] Gaige, A.R., Ayella, A. and Shuai, B. (2010) Methyl Jasmonate and Ethylene Induce Partial Resistance in Medicago truncatula against the Charcoal Rot Pathogen $\mathrm{Ma}$ crophomina phaseolina. Physiological and Molecular Plant Pathology, 74, 412-416. https://doi.org/10.1016/j.pmpp.2010.07.001

[45] Boutrot, F., Segonzac, C., Chang, K.N., Qiao, H., Ecker, J.R., Zipfel, C. and Rathjen, J.P. (2010) Direct Transcriptional Control of the Arabidopsis Immune Receptor FLS2 by the Ethylene-Dependent Transcription Factors EIN3 and EIL1. Proceedings of the National Academy of Sciences of the USA, 107, 14502-14507. 
https://doi.org/10.1073/pnas.1003347107

[46] Sharon, M., Freeman, S. and Sneh, B. (2011) Assessment of Resistance Pathways Induced in Arabidopsis thaliana by Hypovirulent Rhizoctonia spp. Isolates. Phytopathology, 101, 828-838. https://doi.org/10.1094/PHYTO-09-10-0247

[47] Po-Wen, C., Singh, P. and Zimmerli, L. (2013) Priming of the Arabidopsis Pattern-Triggered Immunity Response upon Infection by Necrotrophic Pectobacterium carotovorum Bacteria. Molecular Plant Pathology, 14, 58-70. https://doi.org/10.1111/j.1364-3703.2012.00827.x

[48] Zhu, Z., An, F., Feng, Y., Li, P., Xue, L.A.M., Jiang, Z., Kim, J.M., To, T.K., Li, W., Zhang, X., Yu, Q., Dong, Z., Chen, W.Q., Seki, M., Zhou, J.M. and Guo, H. (2011) Derepression of Ethylene-Stabilized Transcription Factors (EIN3/EIL1) Mediates Jasmonate and Ethylene Signaling Synergy in Arabidopsis. Proceedings of the National Academy of Sciences of the USA, 108, 12539-12544.

https://doi.org/10.1073/pnas.1103959108

[49] O’Donnell, P.J., Calvert, R., Atzorn, R., Wasternack, C., Leyser, H.M.O. and Bowles, D.J. (1996) Ethylene as a Signal Mediating the Wound Response of Tomato Plants. Science, 274, 1914-1917. https://doi.org/10.1126/science.274.5294.1914

[50] Hudgins, J.W. and Franceschi, V.R. (2004) Methyl Jasmonate-Induced Ethylene Production Is Responsible for Conifer Phloem Defense Responses and Reprogramming of Stem Cambial Zone for Traumatic Resin Duct Formation. Plant Physiolo$g y$, 135, 2134-2149. https://doi.org/10.1104/pp.103.037929

[51] Ali, A., Pardo, J.M. and Yun, D.-J. (2020) Desensitization of ABA-Signaling: The Swing from Activation to Degradation. Frontiers in Plant Science, 11, 379.

https://doi.org/10.3389/fpls.2020.00379

[52] Schmidt, K., Pflugmacher, M., Klages, S., Maser, A., Mock, A. and Stahl, D.J. (2008) Accumulation of the Hormone Abscisic Acid (ABA) at the Infection Site of the Fungus Cercospora beticola Supports the Role of ABA as a Repressor of Plant Defense in Sugar Beet. Molecular Plant Pathology, 9, 661-673. https://doi.org/10.1111/j.1364-3703.2008.00491.x

[53] Asselbergh, B., De vleesschauwer, D. and Höfte, M. (2008) Global Switches and Fine-Tuning ABA Modulates Plant Pathogen Defense. Molecular Plant-Microbe Interactions, 21, 709-719. https://doi.org/10.1094/MPMI-21-6-0709

[54] Ton, J., Flors, V. and Mauch-Mani, B. (2009) The Multifaceted Role of ABA in Disease Resistance. Trends in Plant Sciences, 14, 310-317. https://doi.org/10.1016/j.tplants.2009.03.006

[55] Alazem, M., Lin, K.Y. and Lin, N.S. (2014) The Abscisic Acid Pathway Has Multifaceted Effects on the Accumulation of Bamboo Mosaic Virus. Molecular Plant-Microbe Interactions, 27, 177-189. https://doi.org/10.1094/MPMI-08-13-0216-R

[56] El Rahman, T.A., El Oirdi, M., Gonzalez-Lamothe, R. and Bouarab, K. (2012) Necrotrophic Pathogens Use Salicylic Acid Signaling Pathway to Promote Disease Development in Tomato. Molecular Plant-Microbe Interactions, 25, 1584-1593. https://doi.org/10.1094/MPMI-07-12-0187-R

[57] Gimenez-Ibanez, S. and Solano, R. (2013) Nuclear Jasmonate and Salicylate Signaling and Crosstalk in Defense against Pathogens. Frontiers in Plant Science, 4, 72. https://doi.org/10.3389/fpls.2013.00072

[58] Van der Does, D., Leon-Reyes, A., Koornneef, A., Van Verk, M.C., Rodenburg, N., Pauwels, L., Goossens, A., Körbes, A.P., Memelink, J., Ritsema, T., Van Wees, S.C.M. and Pieterse, C.M.J. (2013) Salicylic Acid Suppresses Jasmonic Acid Signaling Downstream of SCF COI1-JAZ by Targeting GCC Promoter Motifs via Tran- 
scription Factor ORA59. The Plant Cell, 25, 744-751.

https://doi.org/10.1105/tpc.112.108548

[59] Zander, M., Chen, S., Imkampe, J., Thurow, C. and Gatz, C. (2012) Repression of the Arabidopsis thaliana Jasmonic Acid/Ethylene-Induced Defense Pathway by TGA-Interacting Glutaredoxins Depends on Their C-Terminal ALWL Motif. Molecular Plant, 5, 831-840. https://doi.org/10.1093/mp/ssr113

[60] Kachroo, A., Lapchyk, L., Fukushige, H., Hildebrand, D., Klessig, D. and Kachroo, P. (2003) Plastidial Fatty Acid Signaling Modulates Salicylic Acid- and Jasmonic Acid-Mediated Defense Pathways in the Arabidopsis ssi2 Mutant. The Plant Cell, 15, 2952-2965. https://doi.org/10.1105/tpc.017301

[61] Leon-Reyes, A., Du, Y., Koornneef, A., Proietti, S., Körbes, A.P., Memelink, J., Pieterse, C.M.J. and Ritsema, T. (2010) Ethylene Signaling Renders the Jasmonate Response of Arabidopsis Insensitive to Future Suppression by Salicylic Acid. Molecular Plant-Microbe Interactions, 23, 187-197.

https://doi.org/10.1094/MPMI-23-2-0187

[62] Spoel, S.H., Koornneef, A., Claessens, S.M.C., Korzelius, J.P., Van Pelt, J.A., Mueller, M.J., Buchala, A.J., Metraux, J.-P., Brown, R., Kazan, K., Van Loon, L.C., Dong, X. and Pieterse, C.M.J. (2003) NPR1 Modulates Cross-Talk between Salicylate- and Jasmonate-Dependent Defense Pathways through a Novel Function in the Cytosol. The Plant Cell, 15, 760-770. https://doi.org/10.1105/tpc.009159

[63] Spoel, S.H., Johnson, J.S. and Dong, X. (2007) Regulation of Tradeoffs between Plant Defenses against Pathogens with Different Lifestyles. Proceedings of the National Academy of Sciences of the USA, 104, 18842-18847. https://doi.org/10.1073/pnas.0708139104

[64] Oliver, R.P. and Solomon, P.S. (2010) New Developments in Pathogenicity and Virulence of Necrotrophs. Current Opinion in Plant Biology, 13, 415-419.

https://doi.org/10.1016/j.pbi.2010.05.003 